\title{
Functional Dysregulation of PBMC and PMN in Crohn's Disease
}

\author{
Saleh A Naser ${ }^{*}$, , Claudia Romero ${ }^{1}$, Sammer Elwasila ${ }^{1}$, Mohamed Ghonaim ${ }^{1}$, Najih Naser $^{2}$ and \\ John F. Valentine ${ }^{3}$ \\ ${ }^{1}$ Department of Molecular Biology and Microbiology. Burnett School of Biomedical Sciences. College of Medicine, \\ University of Central Florida, Orlando, FL 32816, USA \\ ${ }^{2}$ MediSense, Cary, NC, USA \\ ${ }^{3}$ Department of Medicine, University of Florida, Gainesville, Florida, USA
}

\begin{abstract}
Aim: We evaluated the cellular response of polymorphonuclear leukocytes (PMN) and peripheral blood mononuclear cells (PBMC) against viable Mycobacterium avium subspecies paratuberculosis (MAP) for possible understanding of the role of MAP in the pathogenesis of Crohn's disease (CD) patients.

Methods: In vitro phagocytosis and cell proliferation with the aid of Confocal Scanning Laser Microscopy (CSLM) were used to evaluate the immunogenicity of PMN and PBMC freshly obtained from 19 CD patients and 11 healthy controls against viable human-strain MAP, MAP antigens, and Phytohematoagglutonin (PHA), a suspect in CD pathogenesis.

Results: MAP DNA was detected in tissue from 15/17(88\%) CD subjects, $1 / 2(50 \%)$ UC patients and not in blood samples from 11 healthy controls. PMN phagocytosis was suppressed in 13/19 CD subjects (68\%) compared to none in controls ( $\mathrm{p}<0.001)$; suppression ranged from 6 to $97 \%$. PBMC phagocytosis was suppressed only in 5/19 (26\%) of CD subjects; suppression ranged between 1 to $70 \%$. PMN-specific plasma inhibitors were detected in $9 / 19(47 \%)$ CD subjects compared to none in controls $(\mathrm{P}<0.05)$. Plasma inhibitors specific to PBMC phagocytosis were mildly present in $\mathrm{CD}$ patients (3/19 (16\%) CD subjects) compared to none in controls. All $13(68 \%)$ CD subjects that have suppressed PMN phagocytosis were also positive for MAP DNA detection in tissue. PBMC from 8/19 (42\%) CD subjects showed dysfunctional proliferative response against PHA (ranged between 1 to $82 \%$ ) compared to 1 healthy control, suggesting possible T-cell anergy. Out of PBMC from the $11 \mathrm{CD}$ subject's that reacted normally to PHA, 7 reacted strongly to MAP PPD; suggesting pre-exposure to Mycobacteria.. Of 11 healthy control subjects, 10 (91\%) reacted normally to PHA.

Conclusions: It is possible that MAP may directly or indirectly exacerbate a pre-existing defect in phagocyte function - or it may cause the dysfunction employing similar molecular mechanisms used by other pathogenic mycobacteria like $M$. leprae and M. tuberculosis. Collectively the data supports a mycobacterial role in some CD patients.
\end{abstract}

Keywords: Crohn's disease, Mycobacterium avium subsp paratuberculosis, Phagocytosis, Proliferation, PBMC, PMN.

\section{INTRODUCTION}

Crohn's disease (CD) is a chronic inflammatory bowel disease (IBD) of controversial etiology affecting more than 500,000 people in the United States alone [1, 2]. The disease is associated with mouth, larynx, esophagus, stomach, colon, skin, muscle, bone, and synovial tissues tissue [3, 4]. CD has been classified into two distinct disease subtypes: a perforating form and a non-perforating form [5-7]. This spectrum of disease manifestations are similar to bovine paratuberculosis caused by Mycobacterium avium subsp paratuberculosis (MAP). This dual clinical presentation also renders CD analogous to two other mycobacterial diseases, leprosy and tuberculosis [8-10] with attributes of immunoregulatory responses by the immune system [9]. In leprosy, individuals who produce a Th1 biased cell mediated immunity (CMI) reaction develop the contained tuberculoid

*Address correspondence to this author at the Department of Molecular Biology and Microbiology. Burnett School of Biomedical Sciences, BMS 221, College of Medicine. University of Central Florida, Orlando, FL 32816, USA; Tel: (407) 823-0955; Fax: (407) 823-0956;

E-mail: nasers@mail.ucf.edu form of leprosy, while those who produce a Th2 oriented CMI response develop the aggressive lepromatous form of the disease [11]. The role of the Th17 inflammatory pathway in these chronic infectious diseases is some still not fully understood. Some evidence that this type of immunomodulation caused by MAP may exist in CD patients [12]. Mycobacterial heat shock proteins (HSP) are of interest due to their homology with human analogues in addition to their enhanced production of antibodies and $\mathrm{T}$ cells which makes them detectable in patients with $\mathrm{CD}[13,14]$. While pathogenic bacteria such as adherent-invasive $E$. coli have been implicated in the pathogenesis of CD [15], the role of MAP has been alternatively debated. The pathogenesis of MAP would be expected to be similar to M. tuberculosis and M. leprae since they all possess similar molecular components in their cell walls, such as mannose lipoarabinomannan (ManLam). ManLam suppresses antigenic responsiveness of $\mathrm{PBL}$, inhibits antigen presentation, induces production of TNF-alpha and inhibits IFN-gamma mediated macrophage activation [16, 17]. Moreover, significant humoral immune response in $\mathrm{CD}$ patients against MAP was reported [18-21]. The first 
susceptibility gene for CD, NOD2 (nucleotide-binding oligomerization domain), was identified $[22,23]$ and mutations in NOD2 have been shown to be associated with $\mathrm{CD}$, suggesting a role for bacteria-host interactions in the etiology of CD [24]. Other genes such as ATG16L1 and IRGM have also been recently associated with $\mathrm{CD}$ susceptibility to intracellular infections. Bacterial lipoproteins, via toll-like receptors [25] and IFN-gamma released from primed T-cells [26] and NK cells, stimulate macrophages to produce the cytokine IL-12. IL-12 further stimulates primed and naïve Th1 T-cells and also NK cells, inducing the release of TNF. Blocking TNF has been shown to be effective in reducing inflammatory symptoms in moderate to severe CD cases [27]. IL-12 has been proposed as a marker of T-cell activation in sarcoidosis [28] and may be important in mediating the T-cell response in CD [29]. Genetic defects, which result in modified or inactive cytokines or signaling molecules, may also disrupt immune function $[30,31]$. A mutation in the gene of the IFN-gamma signaling chain, which eliminates signaling capability, increases susceptibility to non-tuberculous mycobacterial infection [32]. In vitro stimulation of $\mathrm{T}$ cells with $\mathrm{PPD}$ results in the production of IL-10, IFN-gamma and proliferation in $\mathrm{PPD}(+)$ patients; whereas, cells from anergic patients produce IL-10 but not IFN-gamma and fail to proliferate. Given the similarities between tuberculosis and $\mathrm{CD}$, T-cell anergy as a result of persistent antigen stimulation may be relevant to granuloma formation and inflammation in CD disease. Anergic responses have not yet been investigated in CD. Further demonstration of the mechanisms by which these defects occur will provide valuable insight into the design of more effective treatments for this debilitating inflammatory disease. In this study, we intend to investigate whether the resemblance in immunological events that occur in tuberculosis and CD patients may be related to MAP involvement or not. The main goal is to study the uptake for phagocytosis of MAP by CD patient white blood cells and to stimulate antigenitically blood lymphocytes.

\section{MATERIALS AND METHODOLOGY}

\section{Patient Selection and Specimen Source}

A total of 37 subjects consisting of 23 with $\mathrm{CD}, 3$ with UC, and 11 healthy control individuals were included in this study. Table 1 shows the demographic information and type of specimens obtained from each subject. In order to investigate bacterial infiltration in host tissue, multiple samples of inflamed and non-inflamed tissues and lymph nodes from individual subjects were sought and obtained when possible. Overall, a total of 43 surgically resected tissue specimens were analyzed in this study. Thirty nine tissue samples (17 inflamed, 15 non-inflamed and 7 lymph nodes) were collected from $17 \mathrm{CD}$ subjects, and 4 tissue samples ( 2 inflamed, 1 non-inflamed and 1 lymph node) were collected from two UC patients. Informed consent was obtained from each subject in accordance with Internal Review Board (IRB) regulations at the University of Florida and Gainesville VAMC (Veterans Affair Medical Center). Additionally, a total of 31 peripheral blood samples (19 CD, $1 \mathrm{UC}$, and 11 healthy subjects) were analyzed. A volume of $30 \mathrm{ml}$ of EDTA-whole blood was obtained from each subject when possible and shipped for analysis within 24 hours of collection [33]. Prior to donating clinical specimens, we completed for each subject a survey form with questions concerning family history of IBD, smoking status, ethnic background, fistulizing versus non-fistulizing disease, and medications history, especially of the past 4 weeks (Table 1).

\section{Tissue Collection and Processing}

The tissue samples were catalogued blindly and referred to only by their assigned code. Each tissue was removed from the transport tube and sectioned into three parts. One part was processed for genomic DNA extraction followed by PCR analysis; the second part was homogenized, decontaminated and processed for culture, and the last part was flash frozen in liquid nitrogen.

\section{Peripheral Blood Withdrawal and Processing}

PMN and PBMC were isolated using polymorphoprep and lymphoprep density gradient separation medium following the manufacturer's procedure (Greiner Bio One, Inc). Purified cells were then transferred into sterile tubes for further use.

\section{Microbial Genomic DNA Extraction and PCR Analysis}

The PCR protocol for detection of the MAP IS900 DNA element was performed as described previously [33, 34]. A DNA pellet was dissolved in $250 \mu \mathrm{L}$ TE buffer. The MAP specific IS900 DNA element was evaluated in triplicate using a nested primer PCR technique. Briefly, oligonucleotide primers P90 (5'-GTT-CGG-GGC-CGTCGC-TTA-GG-3') and P91 (5'-GAG-GTC-GAT-CGC-CCACGT-GA-3') were selected to amplify a unique $398 \mathrm{bp}$ fragment of $5^{\prime}$ region of IS900. To reliably detect MAP, AV1(5'-ATG-TGG-TTG-CTG TGT-TGG-ATG-G-3') and AV2(5'-CCG-CCG-CAA-TCA-ACT-CCA-G-3') were used for the nested PCR in the second round using the $398 \mathrm{bp}$ amplified fragment as a template to amplify a 298 bp internal sequence. Representatives of the nested PCR products were gel purified and sequenced by the University of Florida DNA Sequencing Core facility, confirming that the product was identical to the MAP IS900 sequence.

\section{In Vitro Phagocytosis Assay}

MAP was cultured in 7H9 broth media supplemented with mycobactin J (Allied Monitor). Each MAP preparation of $1 \mathrm{x}$ $10^{7} \mathrm{CFU} / \mathrm{ml}$ was labeled by incubating with $100 \mathrm{ug}$ of FITC (Molecular Probes) for $30 \mathrm{~m}$ at room temperature. Following centrifugation at $13,000 \mathrm{rpm}$ for $5 \mathrm{~m}$, the supernatant was discarded, and labeled MAP cells were washed twice with PBS, $\mathrm{pH}$ 7.2. FITC-labeled MAP was then suspended in $100 \mathrm{uL}$ of RPMI media. The efficiency of in vitro phagocytosis of FITClabeled MAP by PMN or PBMC was evaluated as follows. In triplicate, tubes containing $1 \times 10^{6} \mathrm{PMN}$ or PBMC from $19 \mathrm{CD}$ patients, $1 \mathrm{UC}$ patient, and 11 healthy control subjects were incubated with $1 \times 10^{7} \mathrm{CFU}$ of FITC-labeled MAP in the presence of $100 \mathrm{uL}$ plasma from corresponding subject for $2 \mathrm{~h}$ at $37{ }^{\circ} \mathrm{C}$, followed by the addition of $100 \mathrm{uL}$ of $0.2 \%$ trypan blue (Sigma, MO) in order to quench non-specific fluorescence. A volume of $200 \mathrm{uL}$ from each sample was aliquoted into a $96-$ well plate and the absorbance readings were measured at $485 \mathrm{~nm} / 535 \mathrm{~nm}$. A similar set up consisting of PMN, PBMC and 
Table 1. Subjects Demographic Information and Type of Specimens

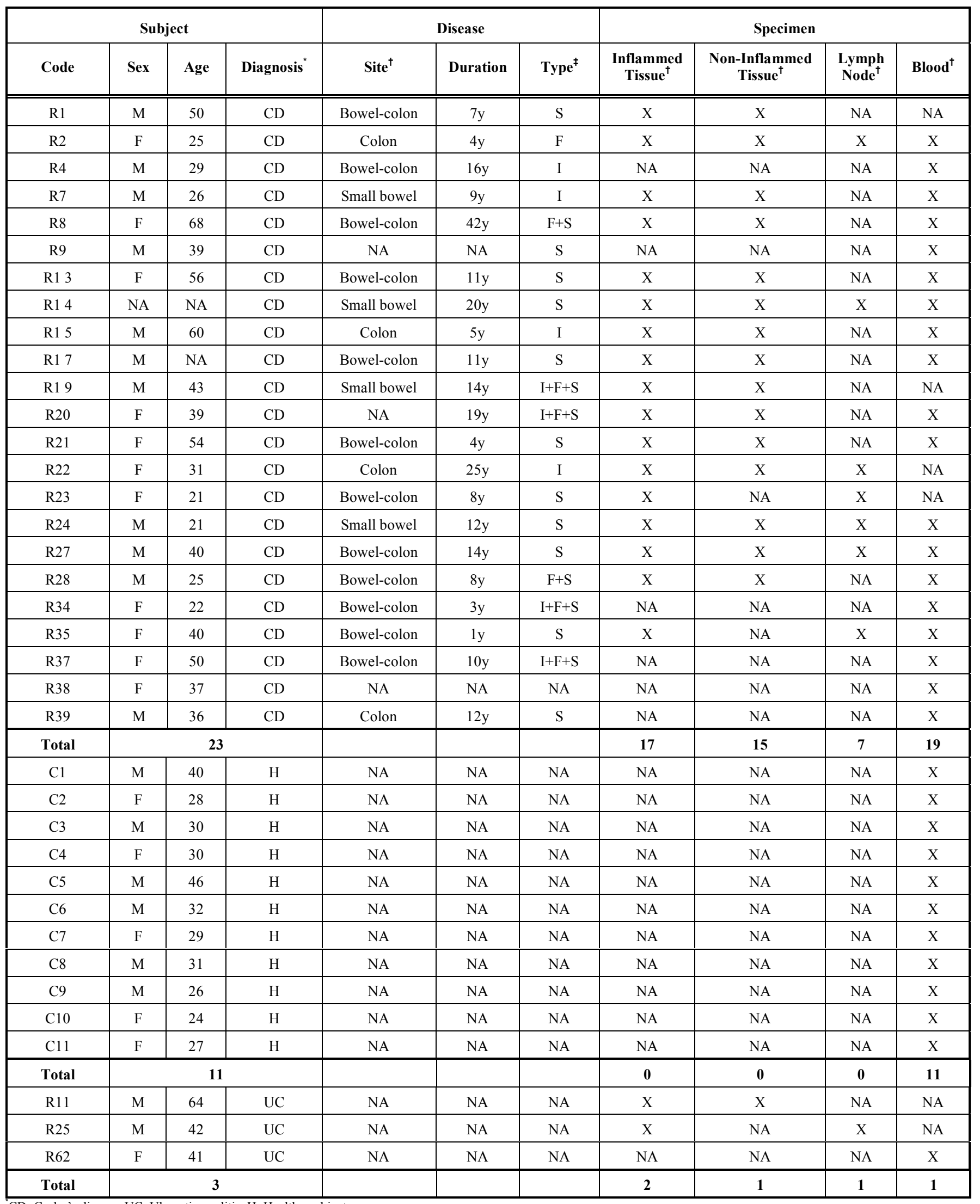

CD: Crohn's disease, UC: Ulcerative colitis, H: Healthy subjects.

${ }^{\dagger} \mathrm{NA}$ : Not not applicable or not available, $\mathrm{X}$ : Specimenspecimen available.

${ }^{\ddagger}$ S: Structuringstructuring state, F: Fistulizingfistulizing state, I: Inflammatoryinflammatory state. 
plasma from a standard control was performed side by side with $\mathrm{CD}$ and healthy control subjects. The amount of internalized FITC-labeled MAP by cells from tested subjects was then compared to that from the standard control. In order to evaluate the presence of plasma inhibitors, crossover experiments were performed where PMN or PBMC from tested subjects were exposed to FITC-labeled MAP in the presence of $100 \mathrm{uL}$ plasma from a standard control and vice versa.

\section{Confocal Scanning Laser Microscopy Analysis}

Three different bacterial strains were used in this study: a clinical MAP strain isolated from CD tissue, M. tuberculosis ATCC \# 25177 (American Type Culture Collection, VA), and Escherichia coli strain TOP10 (Invitrogen, CA). Localization of FITC-labeled viable and dead bacterial cells was investigated in infected PMN by Confocal Scanning Laser Microscope (CSLM). Samples consisting of PMN from $\mathrm{CD}$ and control subjects infected in vitro with FITC labeled-bacterial cells were spotted on microscopic slides, mounted with antifade and scanned by Zeiss CSLM. Slides were scanned under 40x objective lenses with oil. All variables, such as photometric gain, photomultipler setting, pixel size, objective size, mounting medium, and numbers of scans to accumulation were recorded for each scan, and images were documented.

\section{Cell Proliferation Assay}

Initially, the optimum PHA concentration was determined through titration. Aliquots of $1 \times 10^{5}$ cells of PBMC from healthy subjects were plated and exposed to serial dilutions of PHA mitogen at final concentrations of $0.019,0.078,0.31,1.25,5,20$, and $80 \mathrm{ug} / \mathrm{mL}$. The microtiter plates were incubated at $37{ }^{\circ} \mathrm{C}$ and $5 \% \mathrm{CO}_{2}$ for $72 \mathrm{~h}$. PBMC were labeled with bromodeoxyuridine (BrdU, Roche Molecular Biochemicals, IN) and reincubated for additional $24 \mathrm{~h}$. Cell transformation was then measured using a cell proliferation ELISA BrdU commercial kit from Roche (Indianapolis, IN). Immune complexes were detected by a substrate reaction, and the reaction product was quantified by measuring the absorbance at $492 \mathrm{~nm}$ in an ELISA Auto Reader II (Ortho Diagnostic Systems, Inc). Solutions containing optimal concentration of PHA were prepared in sterile RPMI 1640 (Invitrogen, CA). The stocks were dispensed into sterile vials, in suitable volume for one assay, and held frozen at $-20^{\circ} \mathrm{C}$ until use.

Immunoreactivity of PBMC derived from $\mathrm{CD}$ and healthy subjects were evaluated as follows. In triplicate, PBMC at a final dilution of $1 \times 10^{5}$ cells/ well in a 96-well microtiter plate were mixed with plasma from either CD or control subjects. Mitogens and antigens investigated in this study included PHA (20ug/mL, Sigma, MO), Candida albicans extract (20 ug/mL; Greer Laboratories, NC), pokeweed mitogen (PWM, Sigma, MO) at $10 \mathrm{ug} / \mathrm{mL}$ and MAP purified protein derivative (PPD; $5 \mathrm{ug} / \mathrm{mL}$ ). The MAP PPD was prepared in our laboratory by sonication of cell pellets from MAP culture grown to mid-log phase, followed by centrifugation at $16,000 \mathrm{rpm}$ for $30 \mathrm{~m}$. Cell debris was discarded and PPD supernatant was transferred to sterile tubes and stored at $--80{ }^{\circ} \mathrm{C}$ until used. The microtiter plates were incubated at $37{ }^{\circ} \mathrm{C}$ and $5 \% \mathrm{CO}_{2}$ for $72 \mathrm{~h}$. PBMC were labeled with BrdU (Roche Molecular Biochemicals, IN) and reincubated for additional $24 \mathrm{~h}$. Cell transformation was measured in vitro using a cell proliferation ELISA BrdU commercial kit from Roche (Indianapolis, IN) as described earlier.

\section{Statistical Analysis}

Absolute values of one (positive) and zero (negative) were assigned to the observed data for each population and the variance was calculated using the F-test for variances. Data were normalized by multiplying each datum by the inverse of the variance of its group. To evaluate the significance of the differences between each population, a one-way ANOVA was performed on the normalized data. Differences with $\mathrm{p}$ values $<0.05$ were considered significant.

\section{RESULTS}

\section{Detection of IS900 Gene of MAP by Nested PCR}

Two rounds of nested PCR using IS900-derived oligonucleotide primers were employed on 43 DNA extracts isolated directly from homogenized tissue specimens used in this study. Nested PCR detected MAP DNA in 21/43 of all tissue samples, $19 \mathrm{CD}$ tissue and 2 UC tissue. MAP infiltration in host tissue was confirmed following the detection of MAP DNA in neighboring non-inflamed and lymph node tissue. Specifically, MAP DNA was detected in tissue from $15 / 17(88 \%) \mathrm{CD}$ subjects and $1 / 2(50 \%) \mathrm{UC}$ patients. Due to the unavailability of tissue from healthy controls, we tested for the presence of MAP in blood samples provided by these subjects. Consequently, MAP DNA was not detected in any of the healthy controls. As shown in Fig. (1), MAP in $15 \mathrm{CD}$ patients was detected in 9 non-inflamed tissues (Fig. 1A , lanes 2, 7, 11, 13, 20 and Fig. (1B), lanes 4, 7, 10, 13), in 7 inflamed tissues (Fig. (1A), lanes 8, 10, 15, 17, 19, 21 and Fig. (1B), lane 3) and in 4 lymph node tissues (Fig. (1A), lane 26 and Fig. (1B), lanes 2, $5,12)$. In UC patients, MAP DNA was detected in two tissue samples (Fig. (1B), lanes 14 and 15) from 1 out of 2 patients. Following blast and alignment, analysis of the nucleotide sequence amplified from positive tissue samples confirmed the amplification of the IS900 gene unique to MAP.

\section{Evaluation of PMN and PBMC Uptake for Phagocytosis Mechanism}

In vitro phagocytosis of FITC-labeled MAP by PMN and PBMC derived from $19 \mathrm{CD}$ patients was investigated and compared to those from 11 healthy controls. Previously, it was determined that FITC had no toxicity effect on the viability of MAP following a viability study where FITClabeled MAP and unlabeled MAP were inoculated into mycobacterial broth culture media; both treatments gave similar growth properties. The efficiency of phagocytic cells from CD patients to uptake and phagocytose viable FITClabeled MAP was determined by the measurement of internalized fluorescence signal following quenching of unwashed and unphagocytosed FITC-labeled MAP. The uptake of FITC-labeled MAP by subject's PMN or PBMC in the presence of subject's plasma was compared to the uptake of FITC-labeled MAP by PMN or PBMC from a standard normal control in the presence of plasma from the same standard normal control. Table $\mathbf{2}$ summarizes the phagocytosis efficiency of viable MAP by PMN and PBMC and the effect of possible cell-specific plasma inhibitors from blood samples of 30 subjects used in this study. PMN 
(A)

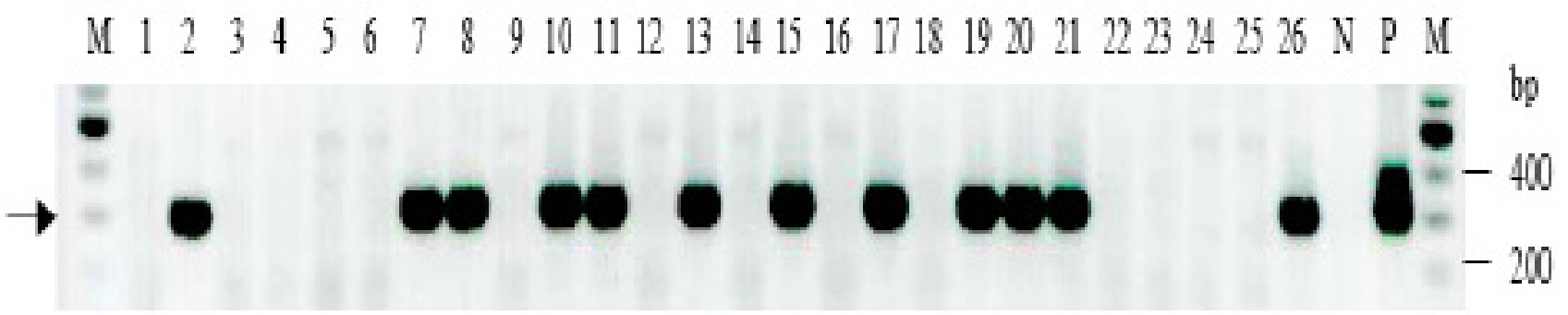

(B)

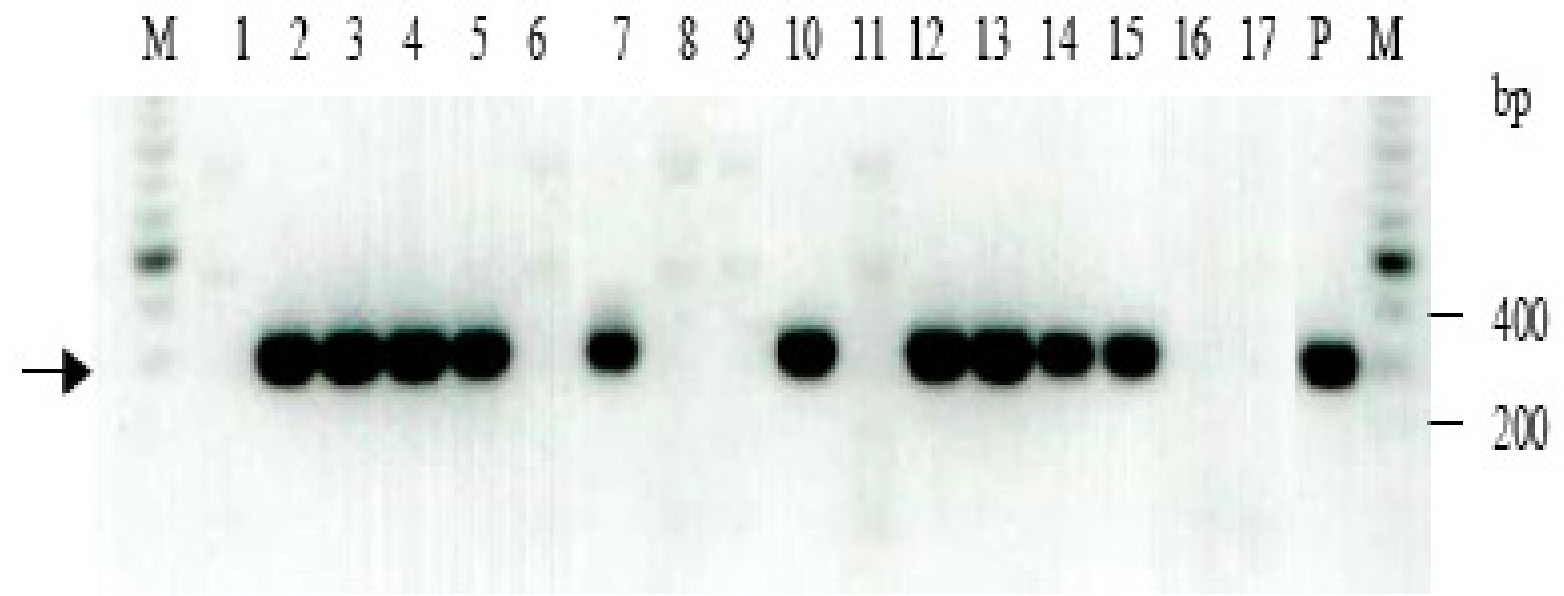

Fig. (1). Nested PCR detection of IS900 DNA Unique to Mycobacterium avium subsp paratuberculosis (MAP) in Surgical Tissue. Forty three DNA extracts isolated from homogenized tissue specimens from $17 \mathrm{CD}$ and 2 UC patients were analyzed by nested PCR. Detection of MAP DNA is indicated by the amplification of a 298 bp fragment. Gel A lanes 2, 4, 7, 9, 11, 13, 16, 18, 20, 22, 24 and gel B lanes 4, 7, 10, 13 represent DNA from non-inflamed tissue from 15 CD patients. Gel A lanes 1, 3, 6, 8, 10, 12, 15, 17, 19, 21, 23, 25 and gel B lanes 1, 3, 6, 9, 11 represent DNA from inflamed tissue from $17 \mathrm{CD}$ patients. DNA from lymph node tissue from $7 \mathrm{CD}$ patients is shown in gel $\mathbf{A}$ lanes 5 , 14, 26 and gel B lanes 2, 5, 8, 12. DNA from non-inflamed tissue (lane B16), two inflamed tissues (lanes B17 and B14) and one lymph node tissue (lane B15) from two ulcerative colitis patients is also shown.

phagocytosis was suppressed in $13 / 19$ CD subjects $(68 \%)$ compared to none in controls $(\mathrm{p}<0.001)$; suppression ranged from 6 to $97 \%$. PBMC phagocytosis was suppressed only in $5 / 19(26 \%)$ of CD subjects; suppression ranged between 1 to $70 \%$. Following exposure of similar amounts of PMN from $\mathrm{CD}$ patients and a standard normal control to $10^{7} \mathrm{CFU}$ of FITC-labeled MAP in the presence of their correspondence plasma, the amount of phagocytosed MAP by PMN was lower in CD patients compared to standard normal control. The quantitative measurement of phagocytosis suppression has been determined for all 31 subjects included in this study (Table 2). Uptake for phagocytosis was evaluated by exposing subject's PMN and PBMC to FITC-labeled MAP in the presence of plasma from the subject compared to a standard healthy control. Percentage of suppression was reported when uptake for phagocytosis by subject's cells was lower than that of the standard healthy control.

A cross over experiment was designed to investigate whether the suppression in uptake for phagocytosis is due to defect in PMN or PBMC, or to the presence of cell-specific inhibitors that may interfere with phagocytosis. Subject's cells incubated with FITC-labeled MAP were tested in the presence of standard control plasma. Accordingly, the uptake value for each cell type may remain similar if cell-specific plasma inhibitors are absent; the opposite is also true. As shown in Table 2, PMN-specific plasma inhibitors were detected in $9 / 19$ (47\%) CD subjects compared to none in controls $(\mathrm{P}<0.05)$. The negative effect of the presence of PMN-specific inhibitors in plasma from $C D$ patients on healthy PMN phagocytosis has also been measured; results ranged between 2 to $67 \%$ (Table 2). Plasma inhibitors specific to PBMC phagocytosis were mildly present in CD patients $(3 / 19(16 \%) C D$ subjects) compared to none in controls. All $13(68 \%)$ CD subjects that have suppressed PMN phagocytosis were also positive for MAP DNA detection in tissue.

The localization of internalized viable and dead bacteria is graphically illustrated in Figs. $(\mathbf{2}, \mathbf{3})$ corresponding to phagocytosis by PMN from healthy and CD subjects respectively. Localization of internalized viable and dead bacteria in UC subjects was found to resemble that of healthy subjects. Viable and dead E. coli (Figs. 2A, 2B, 3A, 3B) and $M$. tuberculosis (Figs. 2C, 2D, 3C, 3D) as well as dead MAP (Figs. 2F, 3F) localized similarly throughout the cytoplasm of PMN from all 3 different subjects. In contrast, viable $M$. tuberculosis and viable MAP localized closer to the cytoplasmic membrane in phagosomes inside PMN isolated only from $\mathrm{CD}$ patients (Fig. 3C, 3E), but not from healthy cells (Fig. 2C, 2E) or UC cells. 
Table 2. In Vitro Evaluation of Phagocytic Uptake of MAP by PMN and PBMC

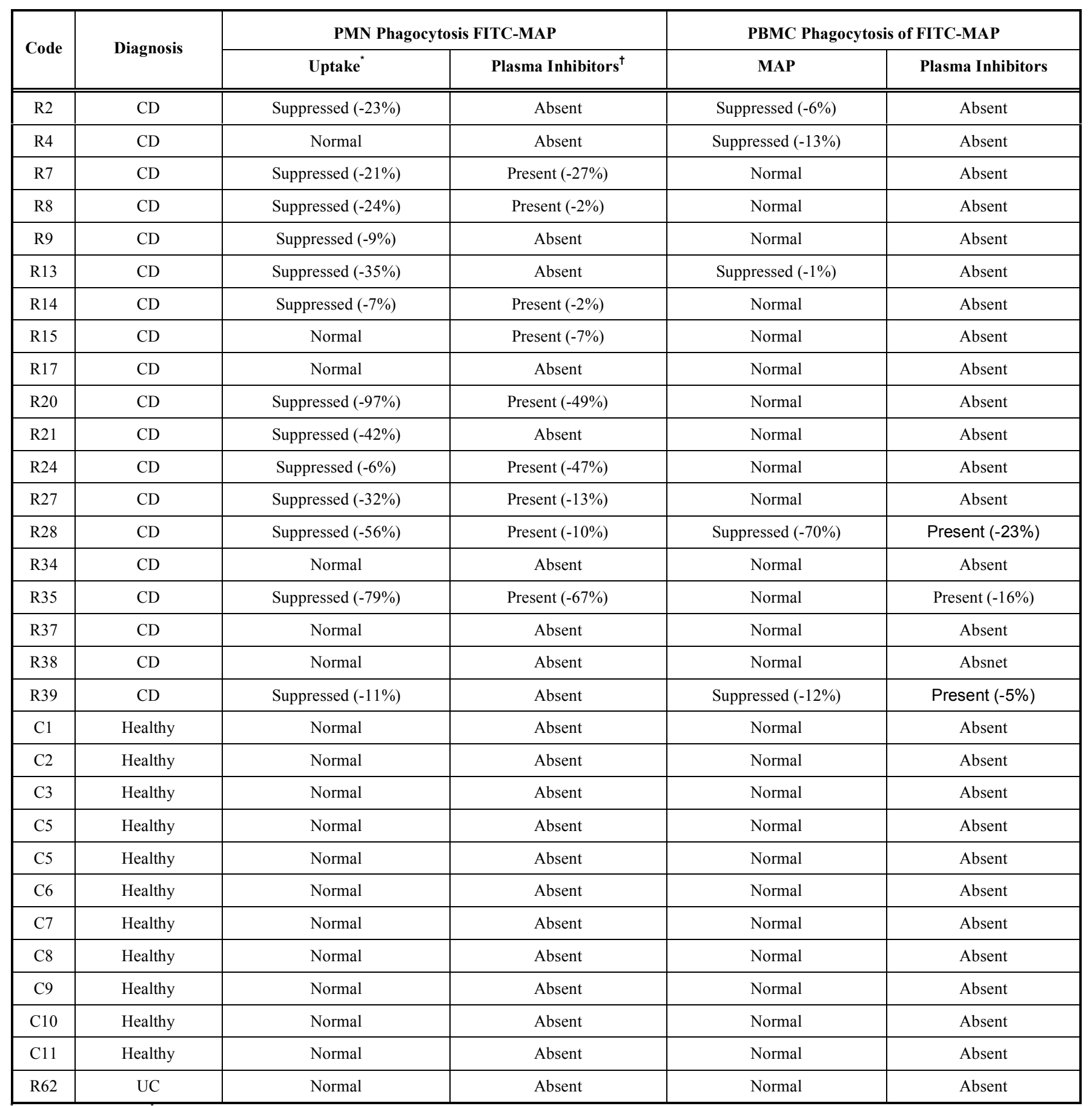

Uptake for hagocytosis Phagocytosis was evaluated by exposing subject's PMN and PBMC to FITC-labeled MAP in the presence of plasma from the subject compared to a standard healthy control. Percentage of suppression was reported when phagocytosis uptake by subject's cells was lower than that of the standard healthy control.

${ }^{\dagger}$ Plasma inhibitors specific for PMN or PBMC phagocytosis were measured by cross-over experiments incubating the plasma from each subject with cells from the standard healthy control and FITC-labeled MAP. These values were compared to those corresponding to the standard healthy control in the presence of plasma without inhibitors.

\section{T-Lymphocyte Proliferation}

In this study, a final concentration of $20 \mathrm{ug} / \mathrm{mL}$ PHA mitogen following $72 \mathrm{~h}$ of incubation was identified to provide optimum lymphocyte activation. Therefore, all experiments including PHA were performed at this PHA concentration. Additionally, the mitogen PWM and extracts from C. albicans and PPD from MAP were used to investigate the proliferative response of T-lymphocytes and their interaction with neighboring relevant cells, such as Blymphocytes and antigen presenting or other dendritric cells. The proliferative response of PBMC isolated from CD and healthy control patients was quantitatively measured and compared to that of PBMC isolated from a standard normal control. Normal proliferative response was reported when 
(A)

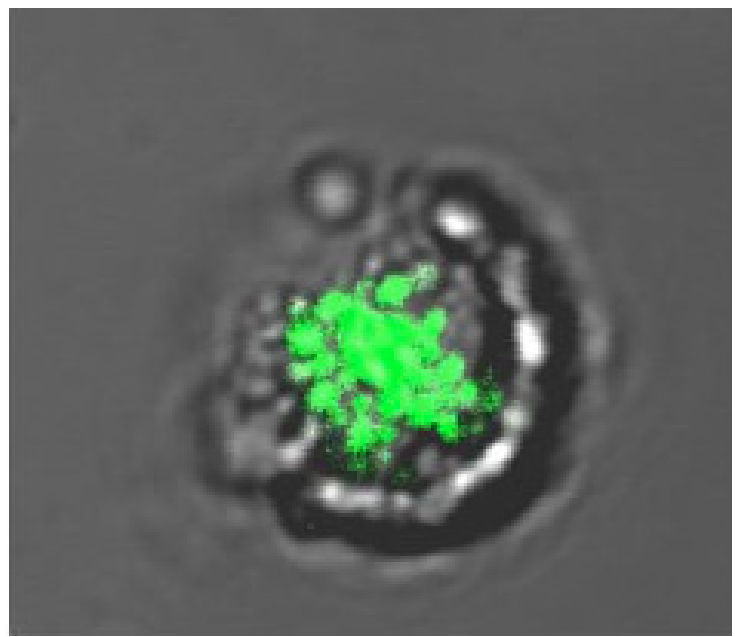

(C)

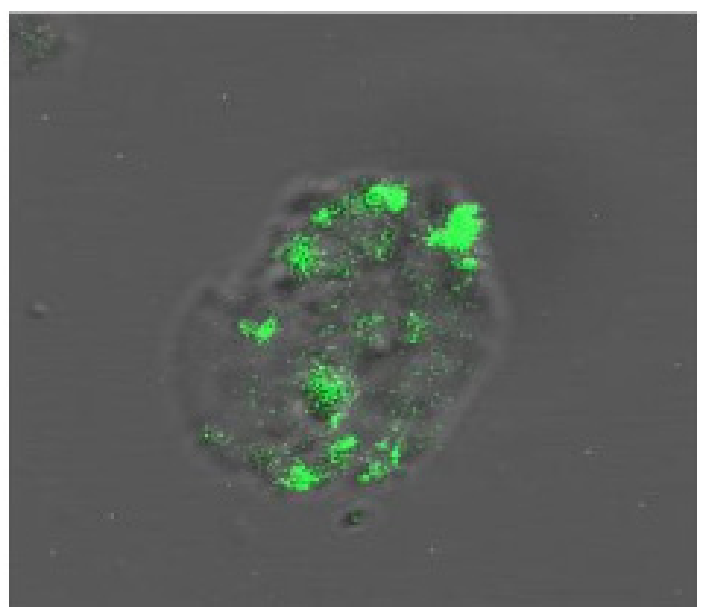

(E)

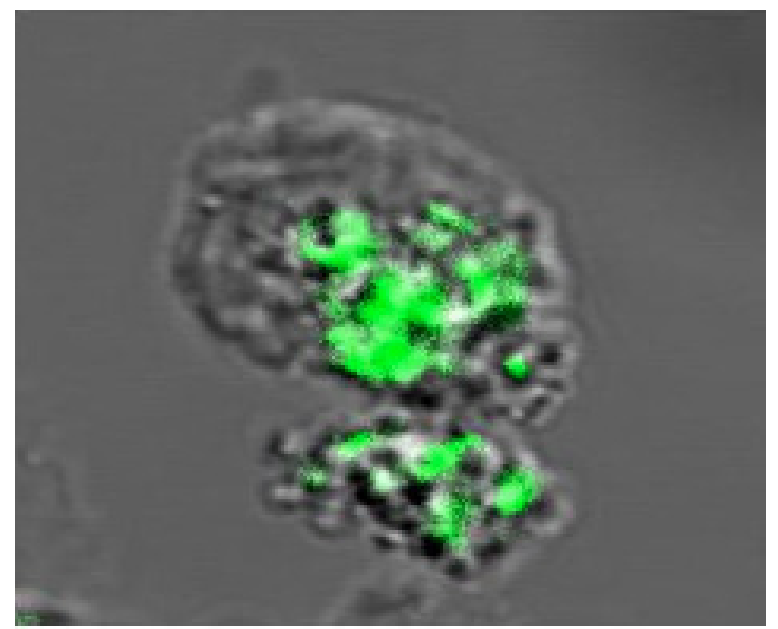

(B)

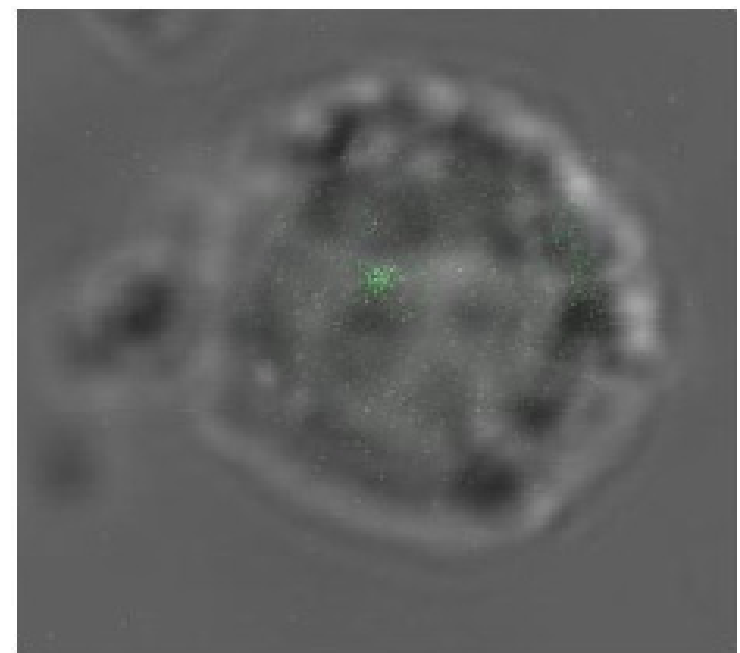

(D)

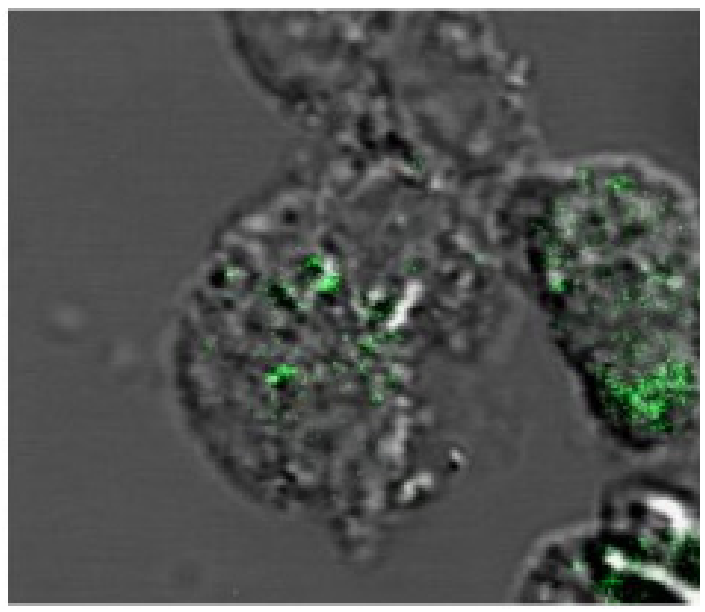

(F)

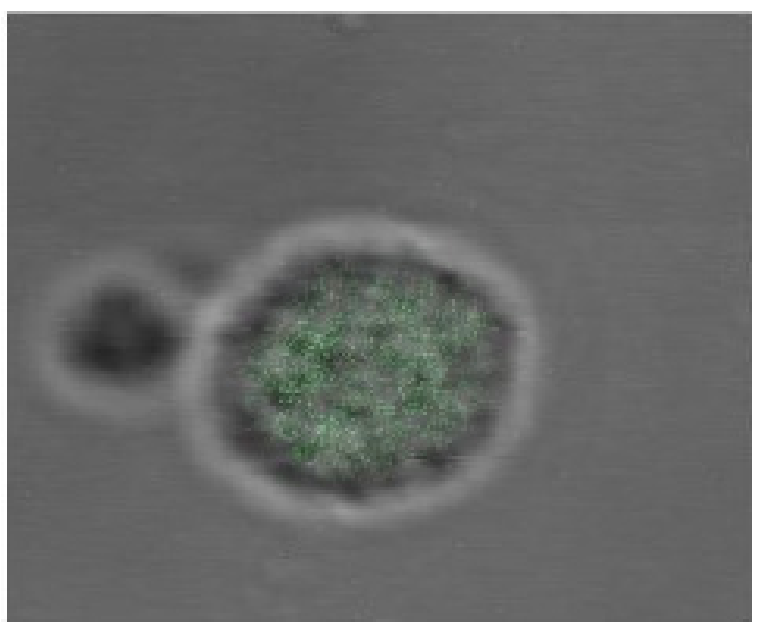

Fig. (2). Confocal Scanning Laser Microscopy (CSLM) Evaluation of the Phagocytosis of FITC-labeled viable and dead bacterial cells by PMN from Healthy subjects. Following two h exposure of PMN to $10^{7} \mathrm{CFU}$ of FITC-labeled viable and dead Escherichia coli (A and B), FITC-labeled viable and dead Mycobacterium tuberculosis (C and D), and FITC-labeled viable and dead MAP (E and F), the amount of phagocytosed bacteria was detected by green fluorescence. Using the Zeiss image analysis software, a threshold was applied to eliminate pixels with no fluorescence (black) and residual noise from the detector. The only fluorescence detected was the one coming from the inside of the phagocytic cells that is directly proportional to the amount of bacterial cells phagocytosed. Viable and dead bacterial cells were localized scattered throughout the cytoplasm inside healthy PMN indicating the desintegration of these bacteria inside the macrophages. 
(A)

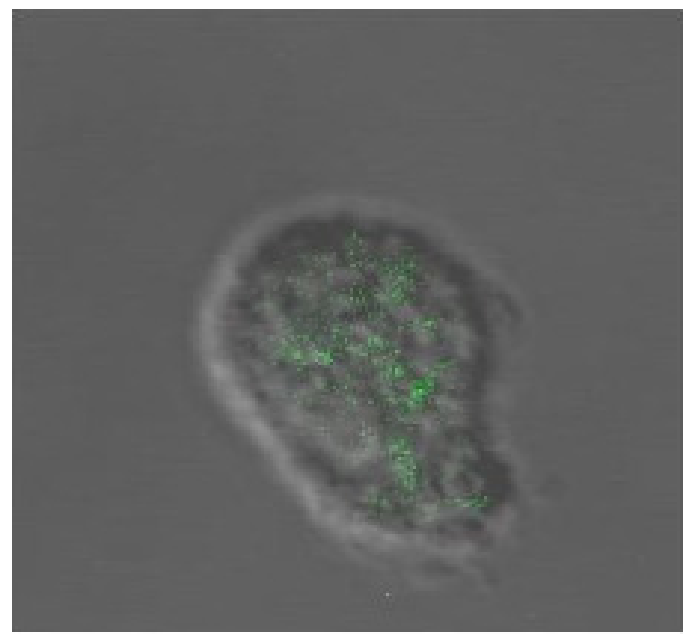

(C)

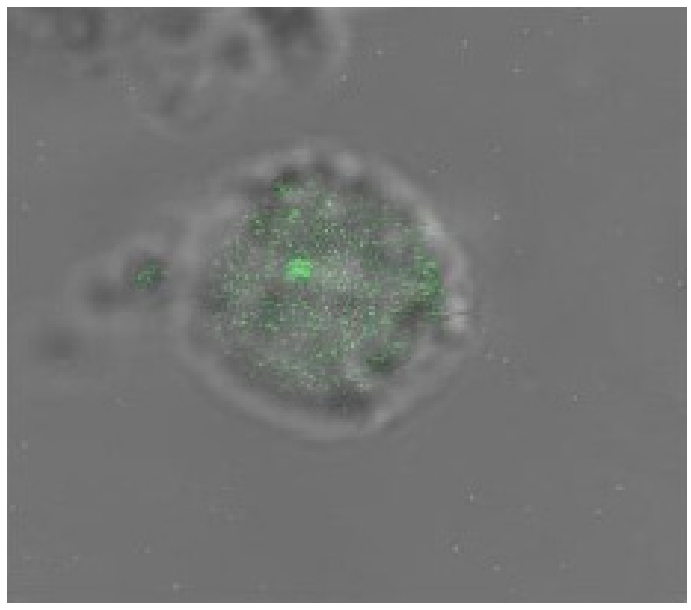

(E)

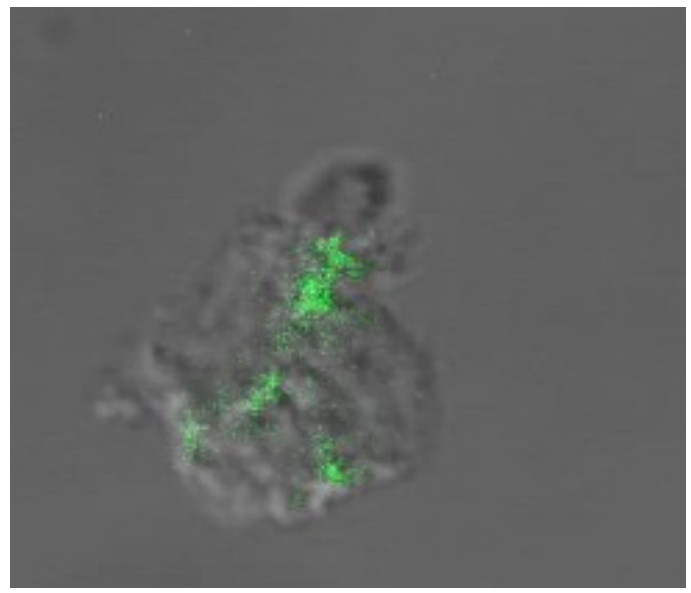

(B)

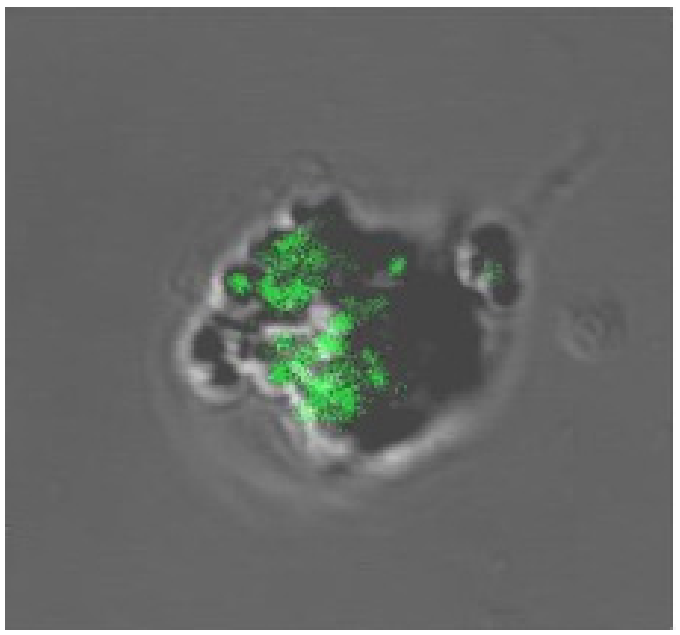

(D)

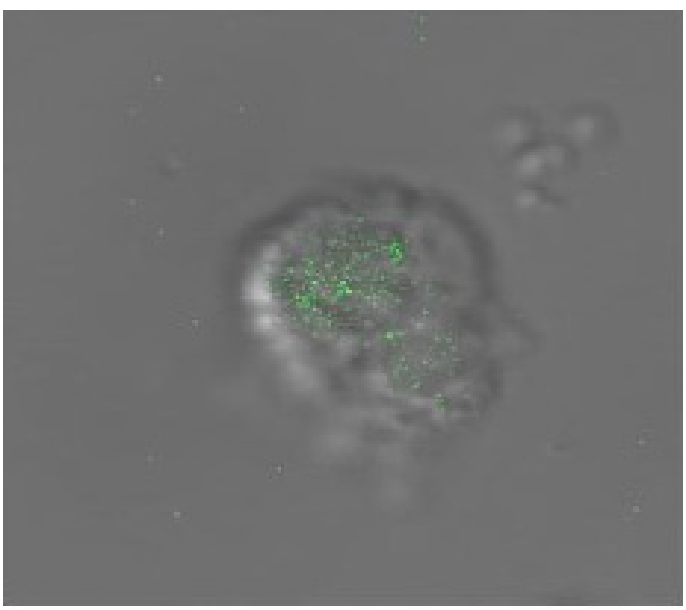

(F)

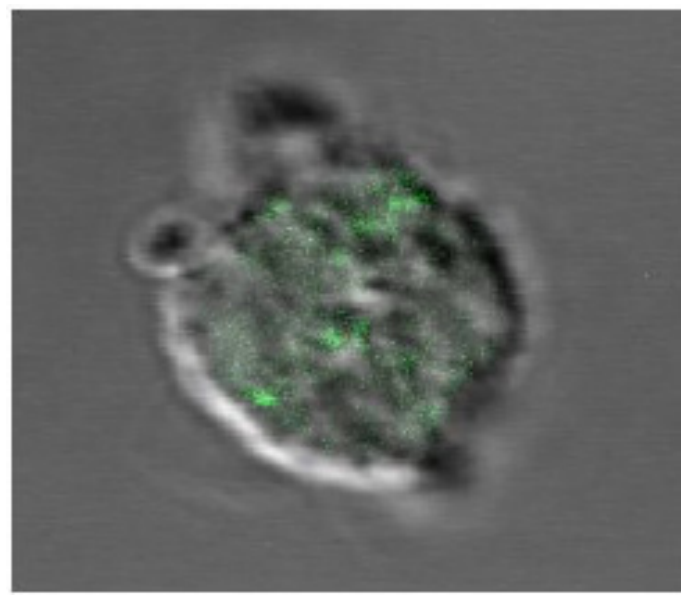

Fig. (3). Confocal Scanning Laser Microscopy (CSLM) Evaluation of the Phagocytosis of FITC-labeled viable and dead bacterial cells by PMN from CD subjects. Following two h exposure of PMN to $10^{7} \mathrm{CFU}$ of FITC-labeled viable and dead Escherichia coli (A and B), FITC-labeled, dead Mycobacterium tuberculosis (C and D), and FITC-labeled viable and dead MAP (E and F), the amount of phagocytosed bacteria was detected by green fluorescence. Using the Zeiss image analysis software, a threshold was applied to eliminate pixels with no fluorescence (black) and residual noise from the detector. The only fluorescence detected was the one coming from the inside of the phagocytic cells that is directly proportional to the amount of bacterial cells phagocytosed. Viable MAP behaved in a way similarly to viable $M$. tuberculosis, where a visible phagocytic vacuole containing bacteria is formed from the membrane $(\mathbf{E})$. 
PBMC from tested subjects matched or reacted better than those from a standard normal control. PBMC from 8/19 (42\%) CD subjects showed dysfunctional proliferative response against PHA (ranged between 1 to $82 \%$ ) compared to 1 control, suggesting possible T-cell anergy. Out of PBMC from the $11 \mathrm{CD}$ subject's that reacted normally to PHA, 7 reacted strongly to MAP PPD; suggesting preexposure to Mycobacteria, one reacted mildly to MAP PPD; suggesting possible cross reactivity with environmental mycobacterial antigens, and 3 did not react with MAP PPD; suggesting lack of Mycobacteria pre-exposure. Of 11 healthy control subjects, $10(91 \%)$ reacted normally to PHA and only $1(9 \%)$ indicated mild T-cell anergy. Mild reactivity to MAP PPD due to cross reactivity with environmental mycobacterial antigens was detected in 2 healthy control subjects. Moreover, PBMC from 3 of these $8 \mathrm{CD}$ subjects reacted negatively against $C$. albicans extract, suggesting also a possible defect in antigen recall ability by activated monocytes. PBMC from the UC patient reacted normally to PHA and slightly reacted to MAP PPD suggesting environmental mycobacterial exposure. Overall, 15 out of 19 (78\%) CD subjects either indicated possible T-cell anergy or MAP pre-exposure compared to detection of MAP DNA in tissue from 15/17 (88\%) CD subjects.

\section{DISCUSSION}

In this study, MAP, which is a controversial etiologic agent of CD, was used to evaluate the phagocytic function of PMN obtained from $\mathrm{CD}$ and UC patients undergoing bowel resections. The results shown in Table 2 reveal a significant decrease in the uptake of FITC-labeled MAP by PMN from $\mathrm{CD}$ subjects when compared to those from controls. This may suggest an original defect in the innate immune response of $\mathrm{CD}$ patients, or it may reflect an acquired, secondary defect in the immune response caused by the disease itself. Phrased more precisely, the compromised phagocytic function may be caused by the etiologic agent (MAP) that causes $\mathrm{CD}$ and thus should be considered an essential part of the pathogenesis of the disease process. Both possibilities may co-exist, especially if a genetic trait predisposes one to an infection with an etiologic microbe. Geijtenbeek et al. [35] demonstrated that M. tuberculosis binds to DC-SIGN (dendritric cell-specific intracellular adhesion molecules (ICAM)) to interfere with DC maturation and signaling that further dysregulates T-cell function and results in an ineffective cell mediated immune response against the bacterium. Localization of MAP near the inner side of the cytoplasmic membrane of an infected PMN from CD patients (Fig. 3E) correlates with a survival mechanism utilized by viable $M$. tuberculosis (Fig. 3C). The data graphically confirms an earlier report which proposed that MAP resists intracellular killing by residing within a phagosomal compartment that retains the characteristics of early phagosomes and resists maturation into functional phagolysosomes [36, 37]. The frequency of detecting MAP DNA in CD tissue as shown in Fig (1) suggests that MAP may be present in the majority of $[38,39]$, and in the blood of at least $50 \% \mathrm{CD}$ patients [34]. This finding suggests that $\mathrm{CD}$ results from a complex interplay of factors including the genetic make-up of the host. As polymorphisms within the NOD2 gene have been associated with increased CD susceptibility [22], other undiscovered genes may be responsible for a genetic defect in PMN phagocytosis of CD patients. This finding is similar to the facts discussed earlier regarding host-agent interaction reported in Brucellosis and tuberculosis diseases. A combination of inadequate clearance of MAP by PMN and impaired macrophage function would be expected to result in granuloma formation.

The effects of MAP infection on phagocytes have been studied in animal models. MAP may be synthesizing biologically active molecules that down-regulate the bactericidal function of macrophages. Similar to other pathogenic mycobacteria, MAP "interferes with normal phagosome- endosome trafficking and interferes with the cooperation of innate and specific immunity. A MAP infection in human Crohn's Disease could affect neutrophil and macrophage function manifesting as impaired phagocytosis.

In lymphocyte derived cells from $\mathrm{CD}$ patients, transformation responses to some antigens assess their degree of prior sensitization. As shown in Table 2, the observation of T-cell anergy in blood samples from significant CD cases compared to none in healthy controls and the fact that T-cells from CD patients have shown to be presensitized to MAP clearly suggest a possible MAP-host interaction.

Given the historical dispute over the etiology and pathogenesis of CD syndrome, the approach of treating the disease by suppressing immune reactions by inflammatory immunosuppressants should be regarded as controversial at best, and detrimental at worst. In contrast, the use of antibiotic regimens for treatment of $C D$ patients has been considered helpful [39-41]. As another approach, the stimulation of the innate immune system with growth factors, including GM-CSF has been proposed [42, 43]. This suggests an immune deficiency in $\mathrm{CD}$ patients, either primary, secondary, or both [44].

\section{CONCLUSION}

The data clearly confirm that 1) MAP is associated with Crohn's Disease, 2) PMN and PBMC phagocytosis is impaired in CD patients, and 3) MAP and M. tuberculosis share similarities in phagosome/endosomal trafficking patterns. It is possible that MAP may directly or indirectly exacerbate a pre-existing defect in phagocyte function - or it may cause the dysfunction employing similar molecular mechanisms used by other pathogenic mycobacteria like $M$. leprae and $M$. tuberculosis. Collectively the data supports a mycobacterial role in some CD patients suggesting that treatment should be directed against this chronic intracellular infection. Overall, the study clearly suggests that a defect in the cellular immune response in $\mathrm{CD}$ patients exposed to MAP perpetuating the disease. The data support a mycobacterial role in at least subsets of CD patients, making treatment with anti-MAP antibiotics desirable.

\section{ACKNOWLEDGEMENTS}

Special thanks to Ms. Heather Hartin for her manuscript editing. This work was supported by grant \# RO1-AI5125101 from NIAID and NIH. 


\section{REFERENCES}

[1] Everhart JE. Digestive diseases in the United States: Epidemiology and impact. NIH Publication 1994; 94 : 1447.

[2] Collins MT, Lisby G, Moser C, et al. Results of multiple diagnostic tests for Mycobacterium avium subsp. paratuberculosis in patients with inflammatory bowel disease and in controls. J Clin Microbiol 2000; 38: 4373-81.

[3] Crohn BB, Ginzburg K, Oppenheimer GD. Regional ileitis: a pathological and clinical entity. JAMA 1932; 99: 1323-9.

[4] Dalziel TK. Chronic Interstitial Enteritis. Br Med J 1913; 2: 1068-70.

[5] Greenstein RJ, Greenstein AJ. Is there clinical, epidemiological, and molecular evidence for two forms of Crohn's Disease?. Mol Med Today $1995 ; 1: 343-8$.

[6] Hermon-Taylor J, Barnes N, Clarke C, Finlayson C. Mycobacterium paratuberculosis cervical lymphadenitis followed five years later by terminal ileitis similar to Crohn's Disease. Br Med J 1998; 316: 449-53.

[7] Mishina D, Katsel P, Brown ST, et al. On the etiology of Crohn disease. Proc Natl Acad Sci USA 1996; 93: 9816-20.

[8] Greenstein AJ, Lachman P, Sachar DB, et al. Perforating and nonperforating indications for repeated operations in Crohn's disease: evidence for two clinical forms. Gut 1988; 29: 588-92.

[9] Vidal PMC, Marques MA, Reddy VM, et al. Systemic dissemination in tuberculosis and leprosy: do mycobacterial adhesins play a role? Microbes Infect 2003; 5(7): 677-84.

[10] Yamamora M, Uyemura K, Deans RJ, et al. Defining protective responses to pathogens: cytokine profiles in leprosy lesions. Science 1991; 254: 277-9.

[11] Britton WJ, Lockwood DN. Leprosy. Lancet 2004; 363(9416): 1209-

[12] Ebert EC, Bhatt BD, Liu S, et al. Induction of suppressor cells by Mycobacterium paratuberculosis antigen in inflammatory bowel disease. Clin Exp Immunol 1991; 83: 320-5.

[13] El-Zaatari FAK, Naser SA, Engstrand L, et al. Nucleotide sequence analysis and seroreactivities of the $65 \mathrm{~K}$ heat shock protein from Mycobacterium paratuberculosis. Clin Diagn Lab Immunol 1995; 2: 657-64.

[14] Markesich DC, Sawai ET, Butel JS, et al. Humoral immune response to stress (Heat Shock) proteins. Dig Dis Sci 1991; 36: 454-60.

[15] Glasser AL, Boudeau J, Barnich N, et al. Adherent Invasive Escherichia coli Strains from patients with Crohn's disease survive and replicate within macrophages without inducing host cell death. Infect Immun 2001; 69(9): 5529-37.

[16] Koets ADP, Rutten VPMG, de Boer M, et al. Differential changes in heat shock protein-, lipoarabinomannan-, and purified protein derivative-specific immunoglobulin G1 and G2 isotype responses during bovine Mycobacterium avium subsp. paratuberculosis infection. Infect Immun 2001; 69: 1492-8.

[17] Mohagheghpour N, van Vollenhoven A, Goodman J, et al. Interaction of Mycobacterium avium with human monocyte-derived dendritic cells. Infect Immun 2000; 68: 5824-9.

[18] El-Zaatari FA, Naser SA, Graham DY. Characterization and seroreactivity of a specific Mycobacterium paratuberculosis recombinant clone expressing $35 \mathrm{~K}$ antigen with clinical and subclinical johne's disease. J Clin Microbiol 1997; 35: 1794-9.

[19] El-Zaatari FAK, Naser SA, Hulten K, et al. Characterization of Mycobacterium paratuberculosis $\mathrm{p} 36$ antigen and its seroreactivities in Crohn's Disease. Curr Microbiol 1999; 39: 115-9.

[20] Naser SA, Shafran I, El-Zaatari FAK. Mycobacterium avium subsp. paratuberculosis in Crohn's disease is serologically positive. Clin Diagn Lab Immunol 1999; 6: 282.

[21] Naser SA, Hulten K, Shafran I, et al. Specific seroreactivity of Crohn's disease patients against p35 and p36 antigens of $M$. avium subsp. paratuberculosis. Vet Microbiol 2000; 77: 497-504.

[22] Ogura Y, Bonen DK, Inohara N, et al. A frameshift mutation in NOD2 associated with susceptibility to Crohn's disease. Nature 2001; 411: 603-6.
[23] Bouma G, Strober W. The immunological and genetic basis of inflammatory bowel disease. Nat Rev Immunol 2003; 3: 521-33.

[24] Girardin SE, Travassos HL, Hervé M, et al. Peptidoglycan molecular requirements allowing detection by Nod1 and Nod2. J Biol Chem 2003; 278: $8869-72$

[25] Hornef MW, Wick MJ, Rhen M, et al. Bacterial strategies for overcoming host innate and adaptive immune responses. Nat Immunol 2002; 3(11): 1033-40.

[26] Camoglio L, Te Velde AA, Tigges AJ, et al. Altered expression of interferon-gamma and interleukin-4 in inflammatory bowel disease. Inflamm Bowel Dis 1998; 4: 285-90.

[27] Sands BE, Anderson FH, Bernstein CN, et al. Infliximab maintenance therapy for fistulizing Crohn's disease. N Engl J Med 2004; 350(9): 876-85.

[28] Kim DS, Jeon YG, Shim TS, et al. The value of interleukin-12 as an activity marker of pulmonary sarcoidosis. Sarcoidosis Vasc Diffuse Lung Dis 2000; 17(3): 271-6.

[29] Pallone F, Monteleone G. Interleukin 12 and Th1 responses in inflammatory bowel disease. Gut 1998; 43: 735-6.

[30] Newport MJ, Huxley CM, Huston S, et al. A mutation in the interferon$\gamma$-receptor gene and susceptibility to mycobacterial infection. N Engl J Med 1996; 335: 1941-9.

[31] Dorman SE, Holland SM. Mutation in the signal -transducing chain of the interferon $-\gamma$-receptor and susceptibility to mycobacterial infection. J Clin Invest 1998; 101(11): 2364-9.

[32] Jouanguy E, Lamhamedi-Cherridi S, Altare F, et al. Partial interferon $\gamma$ - receptor 1 deficiency in a child with tuberculoid bacillus CalmetterGuerin infection and a sibling with clinical tuberculosis. J Clin Invest 1997; 100(11): 2658-64.

[33] Schwartz D, Romero C, Piromalli C, et al. Use of short-term culture for identification of Mycobacterium avium subsp. paratuberculosis in tissue from Crohn's disease patients. Clin Microbiol Infect 2000; 6(6): 303-7.

[34] Naser SA, Ghobrial G, Romero C, et al. Culture of Mycobacterium avium subspecies paratuberculosis from the blood of patients with Crohn's disease. Lancet 2004; 364(9439): 1039-44.

[35] Geijtenbeek TBH, Van Vliet SJ, Koppel EA, et al. Mycobacteria target DC-SIGN to suppress dendritic cell function. J Exp Med 2003; 197: 717.

[36] Kobayashi K, Blaser MJ, Brown WR. Immunohistochemical examination for Mycobacteria in intestinal tissues from patients with Crohn's disease. Gastroenterology 1989; 96(4): 1009-15.

[37] Rumsey J, Valentine JF, Naser SA. Inhibition of phagosome maturation and survival of Mycobacterium avium subspecies paratuberculosis in polymorphonuclear leukocytes from Crohn's disease patients. Med Sci Monit 2006; 12(4): 130-9.

[38] Romero C, Hamdi A, Valentine JF, et al. Evaluation of surgical tissue from patients with Crohn's Disease for the presence of Mycobacterium avium Subspecies paratuberculosis DNA by In Situ hybridization and nested polymerase chain reaction. Inflamm Bowel Dis 2005; 11(2): 116-25.

[39] Naser SA. Mycobacterium in Crohn's disease is hard to digest. Gastroenterology 2005; 129(4): 1360-1.

[40] Shafran I, Kuqler L, El-Zaatari FA, Naser SA, Sandoval J. Open clinical trial of Rifabutin and Clarithromycin in therapy in Crohn's Disease. Dig Liver Dis J 2002; 34(1): 22-8

[41] Chamberlin W, Ghobrial G, Chehtane M, et al. Successful treatment of a Crohn's disease patient infected with bacteremic Mycobacterium paratuberculosis. Am J Gastroenterol 2007; 102(3): 689-91.

[42] Dieckgraefe BK, Korzenik JR. Treatment of active Crohn's disease with recombinant human granulocyte-macrophage colony-stimulating factor. Lancet 2002; 360(9344): 1478-80.

[43] Drumm B, Vaughan D. Granulocyte-macrophage colony-stimulating factor for Crohn's disease. Lancet 2003; 361(9371): 1830

[44] Chamberlin WM, Naser SA. Integrating theories of the etiology of Crohn's disease. On the etiology of Crohn's disease: questioning the hypotheses. Med Sci Monit 2006; 12(2): RA27-33. 